\title{
CAPACIDADE DE COMBINAÇÃO E HETEROSE PARA RESISTÊNCIA A Puccinia polysora UNDERW. EM MILHO
}

\author{
Herberte Pereira da Silva ${ }^{1}$; Mauricio Pires Machado Barbosa²; Luciano Lourenço Nass ${ }^{3}$; Luis \\ Eduardo Aranha Camargo ${ }^{2 *}$ \\ ${ }_{2}^{1}$ Sementes Dow Agrosciences, C.P. 81 - CEP: 14140-000 - Cravinhos, SP. \\ ${ }^{2}$ Depto. de Entomologia, Fitopatologia e Zoologia Agrícola - USP/ESALQ, C.P. 9 - CEP: 13400-970 - Piracicaba, SP. \\ ${ }^{3}$ Embrapa Recursos Genéticos e Biotecnologia, SAIN Parque Rural - CEP: 70770-900 - Brasília, DF. \\ *Autor correspondente <leacamar@carpa.ciagri.usp.br>
}

RESUMO: A resistência genética é o método mais eficiente de controle das doenças foliares da cultura do milho. Para avaliar a capacidade específica e geral de combinação (CEC e CGC, respectivamente) e heterose para resistência a Puccinia polysora, nove linhagens e seus 36 híbridos F1 foram utilizados em experimentos conduzidos em três ambientes. A severidade da doença foi avaliada na planta inteira (PI) e na folha posicionada no ponto de inserção da espiga principal (AFA). O delineamento experimental utilizado foi em blocos casualizados com três repetições e a parcela experimental foi representada por uma fileira de $5 \mathrm{~m}$ de comprimento. A análise da variância para as reações a ferrugem polissora foram feitas usando a análise II do método de Gardner \& Eberhart, associado ao método 4, modelo I de Griffing. A análise dialélica em diferentes ambientes mostrou efeitos altamente significativos $(P<0,01)$ entre ambientes $(E), C G C$ e $C G C \times E$, para os dois métodos de avaliação. O efeito de CEC foi significativo para PI mas não significativo para AFA. A interação CEC x E não foi significativa para os dois métodos de avaliação. A CGC foi mais importante que CEC nas nove linhagens avaliadas, sugerindo que efeitos genéticos aditivos são mais importantes como fonte de variação para resistência a $P$. polysora. Foram encontrados efeitos heteróticos para resistência tanto em cruzamentos entre linhagens resistentes como entre suscetíveis, embora nestes últimos os efeitos tenham sido maiores. Identificaram-se combinações híbridas específicas entre linhagens com alto potencial para o controle genético deste patógeno.

Palavras-chave: análise dialélica, ferrugem polissora, resistência a doença, melhoramento vegetal

\section{COMBINING CAPACITY AND HETEROSIS FOR RESISTANCE TO Puccinia polysora UNDERW. IN MAIZE}

\begin{abstract}
Genetic resistance is the most efficient way of controlling maize leaf diseases. To evaluate the specific and general combining ability (CGE and CGC, respectively) as well as heterosis for resistance to Puccinia polysora, nine inbred lines and a partial diallel set of their $36 \mathrm{~F} 1$ hybrids were used in experiments conducted in three environments. Disease severity was evaluated in the whole plant (PI) and in the leaf positioned at the point of insertion of the main ear (AFA). Trials were conducted in a randomized block design $5 \mathrm{~m}$ row plots. Analyses of variance for disease severity were performed by Gardner and Eberhart's Analysis II, in combination with Griffing's Method 4, Model I. The diallel analysis of environments indicated highly significant environment (E), CGC and CGC x E effects for both evaluation methods; CEC effects significant for PI but not for AFA; interactions CEC x E not significant for both disease severity variables. CGC effects were more important than CEC. This suggests that additive genetic effects are more important as sources of variation for disease resistance in this set of inbred lines. Heterotic effects were found in crossings between resistant lines and between susceptible ones, although in the first case the effects were more evident. Specific hybrid combinations of lines with high potential for the genetic control of this pathogen were identified.
\end{abstract}

Key words: diallel analysis, southern rust, disease resistance, plant breeding

\section{INTRODUÇÃO}

A ferrugem polissora, causada pelo fungo Puccinia polysora Underw. é considerada a mais destrutiva das ferrugens que afetam a cultura do milho. Tem sido relatada em muitas áreas tropicais e subtropicais do mundo onde se cultiva o milho (Melching, 1975; Shurtleff, 1992) e é particularmente bem adaptada a ambientes onde prevalecem temperatura e umidade elevadas. $P$. polysora tem sido considerada de grande importância nas regiões tropicais do Brasil, predominando em regiões abaixo de 700 metros de altitude em semeadura a partir de outubro. Em regiões mais altas, a doença pode ocorrer com maior intensidade geralmente apenas em plantios iniciados a partir de dezembro (Silva, 1997).

A resistência genética é a forma mais eficiente de controle de doenças na cultura do milho (Balmer \& Pereira, 1987; Futrell, 1975), contribuindo para isso a disponibilidade de uma grande diversidade genética. Em 
um programa de melhoramento visando resistência genética a doenças, a determinação dos parâmetros genéticos que governam a resistência permite direcionar os trabalhos de introdução de resistência em germoplasma suscetível e possibilita maiores ganhos de seleção nos métodos a serem empregados. Estimativas da heterose e da capacidade de combinação em cruzamentos entre linhagens permitem determinar a contribuição de cada uma para resistência bem como identificar combinações híbridas de interesse agronômico.

Os conceitos de capacidade geral e específica de combinação (CGC e CEC, respectivamente) têm sido utilizados no melhoramento de várias culturas para produção de grãos, sendo a CGC relativamente mais importante que CEC para linhagens endogâmicas não selecionadas e, ao contrário, menos importante no caso de linhagens previamente selecionadas (Sprague \& Tatum, 1942; Hallauer \& Miranda Filho, 1988; Nass et al., 2000). Estes conceitos são úteis tanto para caracterização de linhagens em cruzamentos como para estabelecer padrões heteróticos entre populações de milho (Beck et al., 1990; Crossa et al., 1990; Han et al., 1991; Hallauer \& Miranda Filho, 1988; Vasal et al., 1992) e têm sido utilizados em estudos da resistência genética a doenças em milho (Lim \& White, 1978; Nelson \& Scott,1973; Callaway et al., 1990).

O presente trabalho teve por objetivos avaliar a capacidade específica e geral de combinação e os efeitos heteróticos na resistência a $P$. polysora em um grupo de linhagens endogâmicas de milho adaptadas às condições do Brasil Central, visando fornecer subsídios para o desenvolvimento de variedades e híbridos resistentes a este patógeno.

\section{MATERIAL E MÉTODOS}

Nove linhagens desenvolvidas pelo Programa de Melhoramento da Companhia Zeneca Sementes Ltda. foram utilizadas para a realização do presente trabalho (TABELA 1). As linhagens foram obtidas através de, no mínimo, sete auto-fecundações sucessivas.

Os campos de cruzamentos para a obtenção dos híbridos simples e multiplicação das linhagens endogâmicas foram instalados na estação experimental da Companhia Zeneca Sementes Ltda, no ano agrícola de 1996/97, em Cravinhos - SP. Todas as linhagens foram intercruzadas utilizando um esquema dialélico completo, obtendo-se assim 36 híbridos simples.

O material vegetal foi avaliado no ano agrícola de 1997/98 na Estação Experimental de Cravinhos - SP, localizada a nordeste do Estado de São Paulo ( $23^{\circ} 20^{\prime} S$ e altitude de 820 metros); numa área experimental da Zeneca Sementes Ltda, em Guaíra-SP, localizada na Região Mogiana do Estado de São Paulo, (22 30 'S sul e altitude de $520 \mathrm{~m}$ ); e na Estação Experimental da Zeneca Sementes Ltda, em Rio Verde-GO, localizada no
TABELA 1 - Linhagens avaliadas para reações a ferrugem polissora e suas origens.

\begin{tabular}{|c|c|c|}
\hline Linhagem & Origem & Reações a $P$. polysora \\
\hline Z1 & Suwan DMR & Resistente \\
\hline Z3 & Suwan DMR & Resistente \\
\hline Z95 & $\begin{array}{l}\text { Sintético Flint } \\
\text { Tropical }\end{array}$ & Resistente \\
\hline Z56 & Suwan DMR & $\begin{array}{l}\text { Moderadamente } \\
\text { Resistente }\end{array}$ \\
\hline Z42 & Suwan DMR & $\begin{array}{l}\text { Moderadamente } \\
\text { Resistente }\end{array}$ \\
\hline Z21 & $\begin{array}{l}\text { Suwan DMR/Flint } \\
\text { Caribe }\end{array}$ & $\begin{array}{l}\text { Moderadamente } \\
\text { Resistente }\end{array}$ \\
\hline Z62 & $\begin{array}{l}\text { Amarillo Dentado/ } \\
\text { Flint Caribe }\end{array}$ & $\begin{array}{l}\text { Moderadamente } \\
\text { Suscetível }\end{array}$ \\
\hline Z072 & $\begin{array}{l}\text { Amarillo Dentado/ } \\
\text { Flint Caribe }\end{array}$ & Suscetível \\
\hline Z93 & BSSS * & Suscetível \\
\hline
\end{tabular}

*BSSS é uma abreviação para lowa Stiff Stalk Synthetic.

sudoeste do Estado de Goiás, (19 15' S e altitude de $700 \mathrm{~m}$ ). As práticas culturais adotadas para o plantio, controle de pragas e condução dos experimentos foram as mesmas nos três locais.

Os ensaios de Cravinhos e Rio Verde foram instalados na primeira quinzena de Dezembro, ao passo que o de Guaíra o foi na segunda quinzena de novembro do mesmo ano agrícola. Um híbrido suscetível foi plantado 30 dias antes do início do experimento e inoculado com uma mistura de urediniosporos oriundos de Rio Verde (GO), Guaíra (SP), Cravinhos (SP) e Campo Mourão (PR). A suspensão do inóculo foi obtida pela lavagem de folhas infectadas. As inoculações foram realizadas aos 30 e 45 dias após o plantio, correspondendo aos estádios de crescimento 3 e 5 , respectivamente, segundo Hanway (1966). A inoculação consistiu na pulverização de cerca de $5 \mathrm{ml}$ da suspensão de esporos no cartucho de cada planta, com um pulverizador BRUDDEN P5-Jr, na concentração de $1 \times 10^{4}$ urediniosporos $\mathrm{mL}^{-1}$ de água destilada, a qual havia sido adicionada uma gota de Tween 80 por $1000 \mathrm{~mL}$.

A severidade da doença foi avaliada na planta inteira (PI) e na folha posicionada no ponto de inserção da espiga principal (AFA) 30 dias após o florescimento, quando as plantas se encontravam aproximadamente no estádio fenológico 7 segundo Fancelli \& Silveira Neto (1997). No primeiro caso foi utilizada a escala diagramática de Rezende et al. (1994) e no segundo a de Fantin (1997).

O delineamento experimental utilizado foi em blocos casualizados com três repetições em todos os ambientes. Dentro de cada repetição, as parcelas úteis de linhagens receberam bordaduras de uma linhagem resistente e as parcelas úteis de híbridos receberam bordaduras de um híbrido simples resistente. A parcela 
experimental foi representada por uma fileira de 5 metros de comprimento, sendo cada planta espaçada $20 \mathrm{~cm}$ de distância, perfazendo uma população de 62.500 plantas ha-1 ${ }^{-1}$ O espaçamento entre fileiras foi de $0,80 \mathrm{~m}$

Dados expressos como notas foram transformados para raiz quadrada e os de porcentagem foram transformados para arco seno da raiz quadrada conforme recomendação de Steel \& Torrie (1960). As análises da variância para as reações à ferrugem polissora foram realizadas de acordo com o método de Gardner \& Eberhart (1966), análise II, associado ao método 4, modelo I de Griffing (1956), para cruzamentos dialélicos completos e experimentos repetidos em vários ambientes (Ferreira et al., 1993). A metodologia é baseada no seguinte modelo matemático.

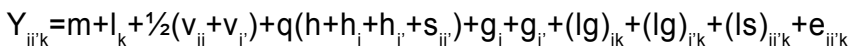
sendo: $Y_{i j i k}=$ a média de todas as repetições do híbrido simples ( $\mathrm{i} \times \mathrm{i}^{\prime}$ ) no ambiente $\mathrm{K}^{\text {th }} ; \mathrm{m}=$ média geral; $\mathrm{I}_{\mathrm{k}}=$ é o efeito ambiental $K^{\text {th }} ; v_{i,} v_{i^{\prime}}=$ efeitos genotípicos das linhagens i e i'; $h=$ heterose média de todos os cruzamentos; hi, hi'=heterose das linhagens i e i'; $g_{i}, g_{i}$ e $s_{i i i=}=$ são os efeitos da capacidade geral e específica de combinação como descrita por Griffing (1956); q=0 para linhagem e q=1 para híbrido; $(\lg )_{\mathrm{ik}^{\prime}},(\mathrm{Ig})_{\mathrm{i}^{\prime} \mathrm{k}^{\prime}},(\mathrm{Is})_{\mathrm{ii} \mathrm{i}^{\prime}}=$ corresponde as interações dos efeitos principais com os ambientes; $e_{i i ' k}=$ é o erro experimental.

\section{RESULTADOS E DISCUSSÃO}

As médias de severidade na planta inteira $(\mathrm{PI}) \mathrm{e}$ na folha da espiga principal (AFA), nos três ambientes, variaram de 2,85 a 9,00 para PI (TABELA 2) e de $1,52 \%$ a $50,00 \%$ (TABELA 3) para AFA. O ambiente de Guaíra foi o que proporcionou os maiores valores médios de severidade da doença obtidos pelos dois métodos de avaliação. As linhagens endogâmicas Z95, Z1 e Z3 e o híbrido Z95 x Z1 apresentaram os menores valores de severidade considerando a média dos três ambientes

TABELA 2 - Médias das reações a P. polysora, avaliadas pela escala de severidade da doença na planta inteira (PI), de nove linhagens endogâmicas e 36 cruzamentos, em três ambientes.

\begin{tabular}{|c|c|c|c|c|c|c|c|c|c|}
\hline \multirow{2}{*}{$\begin{array}{l}\text { Local } \\
\text { Cravinhos }\end{array}$} & \multicolumn{9}{|c|}{ Linhagem } \\
\hline & Z95 & Z1 & Z3 & Z56 & Z42 & Z21 & Z62 & Z072 & Z93 \\
\hline Z95 & 2,99 & 2,85 & 3,28 & 2,97 & 3,04 & 3,06 & 3,32 & 3,85 & 4,36 \\
\hline Z1 & & 3,16 & 2,87 & 3,10 & 3,44 & 3,55 & 3,00 & 3,78 & 2,87 \\
\hline Z3 & & & 3,02 & 3,05 & 3,61 & 3,39 & 3,71 & 3,15 & 3,95 \\
\hline Z56 & & & & 3,47 & 3,29 & 3,87 & 4,39 & 3,63 & 5,38 \\
\hline Z42 & & & & & 3,57 & 4,11 & 3,77 & 3,44 & 5,56 \\
\hline Z21 & & & & & & 3,99 & 3,03 & 3,91 & 3,90 \\
\hline Z62 & & & & & & & 5,19 & 3,72 & 5,52 \\
\hline Z072 & & & & & & & & 5,75 & 5,73 \\
\hline Z93 & & & & & & & & & 5,99 \\
\hline \multicolumn{10}{|l|}{ Gua íra } \\
\hline Z95 & 3,75 & 3,52 & 4,15 & 4,18 & 3,88 & 3,88 & 4,29 & 5,19 & 6,25 \\
\hline Z1 & & 4,59 & 3,91 & 6,17 & 5,36 & 6,12 & 4,44 & 4,78 & 6,78 \\
\hline Z3 & & & 6,24 & 4,19 & 5,66 & 5,12 & 5,40 & 4,58 & 6,50 \\
\hline Z56 & & & & 5,85 & 5,59 & 6,17 & 4,91 & 6,09 & 7,75 \\
\hline Z42 & & & & & 7,24 & 6,66 & 5,49 & 5,90 & 7,75 \\
\hline Z21 & & & & & & 7,05 & 5,72 & 7,12 & 7,38 \\
\hline Z62 & & & & & & & 6,54 & 6,20 & 7,24 \\
\hline Z072 & & & & & & & & 8,74 & 7,05 \\
\hline Z93 & & & & & & & & & 9,00 \\
\hline \multicolumn{10}{|l|}{ Rio Verde } \\
\hline Z95 & 3,55 & 3,10 & 3,88 & 3,83 & 3,41 & 3,47 & 4,36 & 4,85 & 5,61 \\
\hline Z1 & & 4,29 & 3,59 & 4,08 & 4,07 & 3,95 & 4,19 & 4,17 & 6,07 \\
\hline Z3 & & & 5,10 & 4,88 & 3,99 & 4,04 & 5,51 & 4,26 & 6,00 \\
\hline Z56 & & & & & 5,93 & 4,40 & 5,00 & 5,43 & 5,86 \\
\hline Z42 & & & & & 5,10 & 4,14 & 4,41 & 4,35 & 4,96 \\
\hline Z21 & & & & & & 5,52 & 4,57 & 5,54 & 5,21 \\
\hline Z62 & & & & & & & 5,88 & 6,15 & 6,13 \\
\hline Z072 & & & & & & & & 7,14 & 6,74 \\
\hline Z93 & & & & & & & & & 7,60 \\
\hline
\end{tabular}


TABELA 3 - Médias das reações a P. polysora, avaliadas pela porcentagem da área foliar afetada (AFA), de nove linhagens endogâmicas e 36 cruzamentos, em três ambientes.

\begin{tabular}{|c|c|c|c|c|c|c|c|c|c|}
\hline \multirow{2}{*}{$\frac{\text { Local }}{\text { Cravinhos }}$} & \multicolumn{9}{|c|}{ Linhagem } \\
\hline & $Z 95$ & $\mathrm{Z1}$ & Z3 & Z56 & Z42 & Z21 & Z62 & Z072 & Z93 \\
\hline Z95 & 5,94 & 3,30 & 4,92 & 6,96 & 5,15 & 2,30 & 5,82 & 4,57 & 21,99 \\
\hline Z1 & & 5,41 & 1,52 & 5,91 & 11,23 & 8,13 & 4,80 & 6,42 & 23,27 \\
\hline Z3 & & & 8,62 & 6,31 & 9,97 & 6,65 & 11,15 & 6,11 & 15,90 \\
\hline Z56 & & & & 9,76 & 10,09 & 9,05 & 14,43 & 7,87 & 18,74 \\
\hline Z42 & & & & & 21,38 & 15,70 & 7,62 & 6,03 & 42,87 \\
\hline Z21 & & & & & & 6,35 & 3,39 & 7,46 & 10,88 \\
\hline Z62 & & & & & & & 25,12 & 9,63 & 25,53 \\
\hline Z072 & & & & & & & & 18,92 & 22,28 \\
\hline Z93 & & & & & & & & & 32,77 \\
\hline \multicolumn{10}{|l|}{ Gua íra } \\
\hline Z95 & 7,95 & 7,50 & 13,55 & 11,05 & 15,67 & 17,78 & 8,65 & 35,53 & 47,60 \\
\hline Z1 & & 20,52 & 15,93 & 16,68 & 23,19 & 33,27 & 15,05 & 20,27 & 46,23 \\
\hline Z3 & & & 28,15 & 21,75 & 31,19 & 26,92 & 26,64 & 16,55 & 49,62 \\
\hline Z56 & & & & 30,43 & 25,45 & 28,92 & 25,46 & 29,89 & 49,62 \\
\hline Z42 & & & & & 44,68 & 33,52 & 24,06 & 22,85 & 46,99 \\
\hline Z21 & & & & & & 34,28 & 35,22 & 46,65 & 46,82 \\
\hline Z62 & & & & & & & 30,72 & 37,35 & 48,08 \\
\hline Z072 & & & & & & & & 50,00 & 48,47 \\
\hline Z93 & & & & & & & & & 50,00 \\
\hline \multicolumn{10}{|l|}{ Rio Verde } \\
\hline Z95 & 6,56 & 4,00 & 6,15 & 4,94 & 4,24 & 4,03 & 5,70 & 6,60 & 22,94 \\
\hline Z1 & & 9,38 & 7,42 & 7,37 & 9,02 & 9,72 & 8,42 & 7,30 & 24,92 \\
\hline Z3 & & & 22,53 & 15,70 & 12,67 & 7,57 & 21,50 & 7,40 & 36,33 \\
\hline Z56 & & & & 31,87 & 13,00 & 11,38 & 17,65 & 14,27 & 42,29 \\
\hline Z42 & & & & & 30,60 & 9,05 & 13,90 & 7,25 & 15,35 \\
\hline Z21 & & & & & & 20,45 & 5,50 & 14,21 & 17,27 \\
\hline Z62 & & & & & & & 24,52 & 25,00 & 37,73 \\
\hline Z072 & & & & & & & & 36,97 & 43,48 \\
\hline Z93 & & & & & & & & & 50,00 \\
\hline
\end{tabular}

para os dois métodos de avaliação, ao passo que o híbrido Z072 x Z93 foi o mais suscetível. As combinações híbridas com um maior nível de resistência foram obtidas do cruzamento da linhagem Z95 com as linhagens Z1, $Z 3, Z 21$ e Z42 para a variável PI e com as linhagens $Z 1$, $Z 62, Z 21$ e Z3 para a variável AFA, na média das três localidades (TABELAS 2 e 3 ).

A análise dialélica revelou variação significativa entre linhagens para resistência avaliada através dos dois métodos (TABELA 4). Os quadrados médios para heterose total, média, de linhagens e específica também foram significativos para os dois métodos de avaliação, exceto para heterose específica para AFA.

Devido às poucas diferenças entre os métodos de avaliação da doença e a ausência de interação $G \times$ $E$, sugere-se o uso da severidade avaliada na planta inteira (PI) como variável de avaliação por ter apresentado menores coeficientes de variação na análise de variância e por ser de uso mais prático. A utilização de uma única variável reduz o trabalho envolvido com a coleta de dados.
TABELA 4 - Análise conjunta da variância para severidade da doença na planta inteira (PI) e porcentagem da área foliar afetada (AFA) por $P$. polysora para nove linhagens endogâmicas e 36 cruzamentos dialélicos avaliados em três localidades.

\begin{tabular}{|c|c|c|c|}
\hline \multirow[t]{2}{*}{ Fonte de Variação } & \multirow[t]{2}{*}{ G.L } & \multicolumn{2}{|c|}{ Quadrado médio } \\
\hline & & $\mathrm{PI}$ & $\%$ A.F.A. \\
\hline Ambiente $(E)$ & 2 & $0,717^{\star \star}$ & $0,234^{* *}$ \\
\hline Genótipo(corrigido) (G) & 44 & $0,053^{\text {** }}$ & $0,018^{* *}$ \\
\hline Linhagens $(\mathrm{L})$ & 8 & $0,236^{\star *}$ & $0,077^{* *}$ \\
\hline Heterose & 36 & $0,012^{\star *}$ & $0,005^{\star *}$ \\
\hline Heterose Média & 1 & $0,171^{\text {** }}$ & $0,054^{\star \star}$ \\
\hline Heterose Linhagem & 8 & $0,009^{*}$ & $0,006^{\star *}$ \\
\hline Heterose Especifica & 27 & $0,007^{*}$ & $0,003 n . s$ \\
\hline CGC & 8 & $36,603^{* *}$ & $1,384^{* *}$ \\
\hline Interação GxE & 88 & 0,004 n.s & $0,002 n . s$ \\
\hline Erro & 270 & 0,023 & 0,008 \\
\hline $\mathrm{QM}(\mathrm{CGC}) / \mathrm{QM}(\mathrm{CEC})$ & & 5114,670 & 473,740 \\
\hline $\mathrm{CV} \%$ & & 7,036 & 21,271 \\
\hline
\end{tabular}

n.s, ${ }^{*},{ }^{* *}$-Níveis de significância pelo teste $\mathrm{F}$ : não significativo e significativo a $P<0,05$ e $P<0,01$, respectivamente. 
A análise dialélica da capacidade de combinação em diferentes ambientes (TABELA 5) mostrou efeitos altamente significativos $(P<0,01)$ para CGC, ambientes (E) e CGC x E para ambos métodos de avaliação. Também mostrou efeitos significativos $(P<0,05)$ para CEC para resistência avaliada por PI mas não significativos para AFA. Este fato, no entanto, pode ter sido devido a que na avaliação desta última variável considerou-se apenas a área coberta por pústulas e não a posterior necrose decorrente das mesmas, resultando em valores menos elevados de severidade em materiais altamente suscetíveis em comparação à avaliação da PI. $O$ efeito CEC x E não foi significativo para os dois métodos de avaliação. As significâncias dos efeitos do quadrado médio da CGC e CGC x E sugerem a necessidade de seleção de linhas parentais em ambientes específicos. A escolha dos parentais ou genitores baseado na média dos efeitos da CGC pode ser feita se há interesse em híbridos simples adaptados a todos os ambientes. Entretanto, a interação CGC $\times \mathrm{E}$ indica que os maiores valores de CGC não foram os mesmos em todos os ambientes.

Trabalhos prévios para produtividade de grãos e resistência a doenças mostraram que CGC e CEC podem interagir com o ambiente (Rojas \& Sprague, 1952; Matzinger et al., 1959; Parodas \& Hayes, 1971; Pixley \& Bjarnason, 1993; Nelson \& Scott, 1973). Em regiões tropicais, esta interação é de particular interesse pois há uma expressiva variação entre locais, mesmo entre locais próximos. Variações em latitude, comprimento do dia e temperatura são maiores do que em regiões temperadas, o que intensifica o trabalho do melhorista, tornando-o mais desafiador (Miranda Filho, 1985; Paterniani, 1990).

Os efeitos de CGC foram mais importantes que de CEC nesse conjunto de linhagens, a julgar pelos valores de seus quadrados médios. Assim, depreendese que efeitos genéticos aditivos são mais importantes como fonte de variação para resistência a esta doença avaliada por ambos métodos (TABELA 5). Estes resultados estão de acordo com os obtidos por Fantin et al. (1993) onde os autores, estudando a variação entre gerações segundo o modelo de Mather \& Jinks (1971), observaram, em cruzamento de uma linhagem resistente com uma suscetível e gerações derivadas (F1, F2 e retrocruzamentos), maior importância de efeito aditivo que dominante como fonte de variação para resistência à $P$. polysora. Conseqüentemente, deve-se dar ênfase ao desempenho médio da linhagem nas combinações híbridas durante a seleção. Combinações específicas significativas também foram encontradas, embora a um menor nível de significância. Se a CEC também é importante na expressão da resistência a doença mesmo que somente em alguns cruzamentos, seus efeitos, se positivos ou negativos, deverão ser consistentes nos ambientes haja vista a não significância do termo CEC $\times$ E.
TABELA 5 - Quadrados médios da capacidade geral de combinação e capacidade específica de combinação e das interações com locais para severidade na planta inteira $(\mathrm{PI})$ e porcentagem da área foliar afetada (AFA) por P. polysora para nove linhagens endogâmicas e 36 cruzamentos dialélicos avaliados em três localidades.

\begin{tabular}{lccc}
\hline Fonte de Variação & G.L & \multicolumn{2}{c}{ Quadrado médio } \\
\hline & & $\mathrm{PI}$ & $\% \mathrm{~A} . \mathrm{F} . \mathrm{A}$. \\
Ambiente $(\mathrm{E})$ & 2 & $13,733^{* *}$ & $0,234^{* *}$ \\
CGC & 8 & $36,603^{* *}$ & $1,384^{* *}$ \\
CEC & 27 & $0,007^{*}$ & 0,003 n.s. \\
CGC x E & 8 & $73,936^{* *}$ & $3,066^{* *}$ \\
CEC $\times$ E & 27 & 0,025 n.s. & 0,012 n.s \\
Erro combinado & 270 & 0,023 & 0,008 \\
\hline CV \% & & 7,036 & 21,271 \\
\hline
\end{tabular}

n.s, ${ }^{*},{ }^{* *}$-Níveis de significância pelo teste F: não significativo e significativo a $\mathrm{P}<0,05$ e $\mathrm{P}<0,01$, respectivamente.

TABELA 6 - Estimativas médias da capacidade geral de combinação(gi) para escala de severidade da doença na planta inteira $(\mathrm{PI})$ e porcentagem da área foliar afetada (AFA) das reações a $P$. polysora, de acordo com o método de Gardner e Eberhart, análise II, associado ao método 4 de Griffing (1956) modelo I, para nove linhagens em três locais.

\begin{tabular}{|c|c|c|}
\hline $\begin{array}{l}\text { Linhagem } \\
\text { end ogâmica }\end{array}$ & \multicolumn{2}{|c|}{$\begin{array}{l}\text { Capacidade geral de } \\
\text { combinação (gi) }\end{array}$} \\
\hline & Severidade da doença & A.F. A. \\
\hline Z95 & $-0,587$ & $-0,353$ \\
\hline Z 1 & $-0,416$ & $-0,217$ \\
\hline Z 3 & $-0,308$ & $-0,091$ \\
\hline Z56 & 0,028 & $-0,019$ \\
\hline Z42 & $-0,067$ & $-0,014$ \\
\hline Z21 & 0,015 & $-0,042$ \\
\hline Z62 & 0,097 & 0,024 \\
\hline Z072 & 0,254 & 0,035 \\
\hline Z93 & 0,985 & 0,675 \\
\hline Desvio padrão $\left(g_{i}\right)$ & 0,173 & 0,107 \\
\hline Desvio padrão $\left(g_{i}-g_{j}\right)$ & 0,243 & 0,151 \\
\hline
\end{tabular}

Estimativas médias da CGC, de três ambientes, para as nove linhagens avaliadas pelos dois métodos são apresentadas na TABELA 6. As direções dos efeitos genéticos para resistência a doença foram negativas em vários casos porque plantas resistentes apresentaram menores severidades. Metade das linhagens parentais, avaliadas através dos dois métodos, apresentaram efeitos de CGC negativos, indicando que, na média, estes parentais contribuíram para aumentar a resistência em cruzamentos. As linhagens Z95 e Z93 apresentaram os maiores efeitos negativos e positivos de CGC, respectivamente, para os dois métodos de avaliação, sendo que os efeitos altamente significativos e negativos da CGC foram encontrados para as linhagens Z95, Z1 e Z3, para os dois métodos de avaliação e para os cruzamentos entre as linhagens resistentes. Efeitos heteróticos para 
TABELA 7 - Estimativas médias da capacidade específica de combinação(s $\left.\mathrm{s}_{\mathrm{ij}}\right)$ para severidade da doença na planta inteira (PI) (diagonal acima) e porcentagem da área foliar afetada (AFA) (diagonal abaixo) por P. polysora, de acordo com o método de Gardner e Eberhart, análise II, associado ao método 4 de Griffing (1956) modelo I, para 36 cruzamentos dialélicos em três locais.

\begin{tabular}{lcccccrrrc}
\hline & Z95 & Z1 & Z3 & Z56 & Z42 & Z21 & Z62 & Z072 & Z93 \\
\hline Z95 & - & $-0,038$ a & 0,093 & $-0,051$ & $-0,073$ & $-0,095$ & 0,011 & 0,112 & 0,042 \\
Z1 & $-0,017$ b & - & $-0,049$ & $-0,059$ & 0,075 & 0,102 & $-0,076$ & $-0,037$ & 0,082 \\
Z3 & 0,014 & $-0,041$ & - & $-0,051$ & 0,070 & $-0,011$ & 0,125 & $-0,136$ & $-0,41$ \\
Z56 & $-0,039$ & $-0,026$ & 0,006 & & $-0,042$ & 0,069 & 0,026 & 0,025 & 0,083 \\
Z42 & $-0,017$ & 0,044 & 0,045 & 0,004 & & 0,085 & $-0,028$ & $-0,087$ & $-0,002$ \\
Z21 & $-0,021$ & 0,081 & $-0,008$ & 0,013 & 0,047 & & $-0,093$ & 0,096 & $-0,154$ \\
Z62 & $-0,043$ & $-0,044$ & 0,064 & 0,032 & $-0,027$ & $-0,054$ & & 0,035 & $-0,001$ \\
Z072 & 0,060 & $-0,022$ & $-0,086$ & $-0,003$ & $-0,085$ & 0,060 & 0,065 & & 0,035 \\
Z93 & 0,064 & 0,027 & 0,006 & 0,013 & $-0,011$ & $-0,118$ & 0,007 & 0,012 & - \\
\hline
\end{tabular}

a. Desvio padrão. $\left(\mathrm{s}_{\mathrm{ij}}\right)=0,065 ;\left(\mathrm{s}_{\mathrm{ij}}-\mathrm{s}_{\mathrm{ik}}\right)=0,098 ;\left(\mathrm{s}_{\mathrm{ij}}-\mathrm{s}_{\mathrm{k})}\right)=0,090$

b. Desvio padrão. $\left(s_{i j}\right)=0,041 ;\left(s_{i j}-s_{i k}\right)=0,062 ;\left(s_{i j}-s_{k l}\right)=0,056$

resistência foram encontrados tanto em cruzamentos entre linhagens resistentes como entre linhagens suscetíveis, sendo que nestes últimos os efeitos heteróticos foram maiores.

O maior efeito da CEC negativa e positiva para a avaliação da severidade da doença na planta inteira (PI) foi observado nos cruzamentos Z21 x Z93 e Z3 x Z62, respectivamente. (TABELA 7). Para a avaliação através da AFA, o maior efeito da CEC negativa e positiva foi observado no cruzamento das linhagens Z21 x Z93 e Z21 x Z1, respectivamente. Estes resultados ilustram a magnitude dos efeitos da CEC em combinações híbridas para resistência a esta doença, combinações estas que podem ser utilizadas com sucesso no controle genético desta doença.

\section{REFERÊNCIAS BIBLIOGRÁFICAS}

BALMER, E.; PEREIRA, O.A.P. Doenças do milho. In: PATERNIANI, E.; VIEGAS, G.P. (Ed.) Melhoramento e produção de milho. 2.ed. Campinas: Fundação Cargill, 1987. v.2, cap.14, p.595-634.

BECK, D.L.; VASAL, S.K.; CROSSA, J. Heterosis and combining ability of CIMMYT's tropical early and intermediate maturity maize (Zea mays L.) germplasm. Maydica, v.35, p.279-285, 1990.

CALLAWAY, M.B.; SMITH, M.E.; COFFMAN, W.R. Diallel analysis of resistance to anthracnose stalk rot in maize inbreds. Crop Science, v.30, p.335-337, 1990.

CROSSA, J.; VASAL, S.K.; BECK, D.L. Combining ability estimates of CIMMYT's tropical late yellow maize germplasm. Maydica, v.35, p.273-278, 1990.

FANCELLI, L.C.; SILVEIRA NETO, A. Aspectos fisiológicos da cultura do milho. In: SIMPÓSIO SOBRE A CULTURA DO MILHO, Piracicaba, 1997. Anais. Piracicaba: ESALQ/USP, 1997. p.60-67

FANTIN, G.M.; SILVA, H.P.; BALMER, E.; MIRANDA FILHO, J.B. de. Herança da resistência à ferrugem causada por Puccinia polysora em milho. Fitopatologia Brasileira, v.18, p.332-333, 1993.

FANTIN, G.M. Avaliação de resistência do milho a ferrugem causada por Puccinia polysora UNDERW. Piracicaba, 1997. 136p. Tese (Doutorado) - Escola Superior de Agricultura "Luiz de Queiroz", Universidade de São Paulo.
FERREIRA, D.F.; REZENDE, G.D.S.P.; RAMALHO, M.A.P. An adaptation of Griffing's method IV of complete diallel cross analysis for experiments repeated in several environments. Brazilian Journal of Genetics, v16, p.357366, 1993.

FUTRELL, M.C. Puccinia polysora epidemics on maize associated with cropping practice and genetic homogeneity. Phytopathology, v.65, p.1040-1042, 1975.

GARDNER , C.O.; EBERHART, S.A. Analysis and interpretation of the variety cross diallel and related populations. Biometrics, v.22, p.439-452, 1966.

GRIFFING, B. Concept of general and specific combining ability in relation to diallel crossing systems. Australian Journal of Biological Science, v.9, p.463-493, 1956.

HALLAUER, A.R.; MIRANDA FILHO, J.B. Quantitative genetics in maize breeding. Ames: lowa State University Press, 1988. 375p.

HAN, G.C.; VASAL, S.K.; BECK, D.L.; ELIAS, E. Combining ability of inbred lines derived from CIMMYT maize (Zea mays L.) germplasm. Maydica, v.36, p.57-64, 1991.

HANWAY, A.L. How a corn plant develops. Special Reporter lowa Agricultural Experimental Station, v.48, p.4-15, 1966.

LIM, S.M.; WHITE, D.G. Estimates of heterosis and combining ability for resistance of maize to Colletotrichum graminicola. Phytopathology, v.68, p.1336-1342, 1978.

MATHER, K.; JINKS, J.L. Biometrical genetics. Ithaca: Cornell University Press, 1971. 382p.

MATZINGER, D.F.; SPRAGUE, G.F.; COCKERHAM, C.C. Diallel cross of maize in experiments repeated over locations and years. Agronomy Journal, v.51, p.346-350, 1959.

MELCHING, J.S. Corn rusts: types, races and destructive potencial. In: ANNUAL CORN AND SORGHUM RESEARCH CONFERENCE, 30., Washington, 1975. Washington: American Seed Trade Association, 1975. p.90-115.

MIRANDA FILHO, J.B. Breeding methodologies for tropical maize. In: BRANDOLINI, A.; SALAMINI, F. (Ed.) Breeding strategies for maize production improvement in the tropics. Firenze: FAO; Instituto Agronomico per l'Oltremare, p.350. 1985.

NASS, L.L.; LIMA, M.; VENCOVSKY, R.; GALLO, P.B. Combining ability of maize inbred lines evaluated in three environments in Brazil. Scientia Agricola, v.57, p.129-134, 2000.

NELSON, L.R.; SCOTT, G.E. Diallel analysis of resistance of corn (Zea mays L.) to Corn Stunt. Crop Science, v.13, p.162164, 1973. 
PARODAS, R.S.; HAYES, J.D. An investigation of genotypeenvironment interactions for rate of ear emergence in spring barley. Heredity, v.26, p.157-175, 1971.

PATERNIANI, E. Maize breeding in the tropics. CRC Critical Reviews in Plant Science, v.9, p.125-154, 1990.

PIXLEY, K.V.; BJARNASON, M.S. Combining ability for yield and protein quality among modified-endosperm opaque-2 tropical maize inbreds. Crop Science, v.33, p.1229-1234,1993.

REZENDE, I.C.; SILVA, H.P.; PEREIRA, O.A.P. Perdas da produção de milho causada por Puccinia polysora Underw. In: CONGRESSO NACIONAL DE MILHO E SORGO, 20., Goiania, 1994. Resumos. Goiania: ABMS, 1994.

ROJAS, B.A.; SPRAGUE, G.F. A comparision of variance components in corn yield trials: III. General and specific combining ability and their interaction with locations and years. Agronomy Journal, v.44, p.462-466, 1952.

SHURTLEFF, M.C. (Ed.) Compendium of corn diseases. 2.ed. St. Paul: American Phytopathological Press, 1992.105p.
SILVA, H.P. Incidência de doenças fúngicas na "safrinha". In: SEMINÁRIO SOBRE A CULTURA DO MILHO "SAFRINHA", 4., Assis, 1997. Anais. Campinas: IAC, CDV, 1997. p.81-86. SPRAGUE, G.F.; TATUM, L.A. General vs. specific combining ability in single crosses of corn. Journal of the American Society of Agronomy, v.34, p.923-932, 1942.

STEEL, R.G.D.; TORRIE, J.H. Principles and procedures of statistics. New York: McGraw Hill, 1960. 481p.

VASAL, S.K.; SRINIVASAN, G.; HAN, G.C.; GONZALES, C.F. Heterotic patterns of eighty-eight white subtropical CIMMYT maize lines. Maydica, v.37, p.319-327, 1992.

Recebido em 29.01.01 